\title{
Thermisch induzierter Abbau von Disaccharid-Amadori-Verbindungen in quasi wasserfreiem Reaktionsmilieu
}

\author{
Lothar Kroh $^{1}$, Ronald Schrödter ${ }^{2}$, Clemens Mügge ${ }^{1}$, Günther Westphal ${ }^{1}$ und Werner Baltes ${ }^{2}$ \\ 1 Humboldt-Universität zu Berlin, Institut für Lebensmittelwissenschaft und Mikrobiologie und Institut für Analytische Chemie, \\ Invalidenstrasse 42, O-1040 Berlin, Bundesrepublik Deutschland \\ 2 Technische Universität Berlin, Institut für Lebensmittelchemie, Gustav-Meyer-Allee 25, W-1000 Berlin 65, Bundesrepublik Deutschland
}

Wir möchten unsere Leser darauf aufmerksam machen, daß der Name von Herrn Ronald Schrödter unglücklicherweise in dem oben genannten Heft falsch abgedruckt wurde. 